\title{
Structure inhomogeneities, shallow defects, and charge transport in the series of thermoelectric materials $\mathrm{K}_{2} \mathrm{Bi}_{8-x} \mathrm{Sb}_{x} \mathrm{Se}_{13}$
}

\author{
Theodora Kyratsi \\ Department of Mechanical and Manufacturing Engineering, University of Cyprus, 1678 Nicosia, Cyprus
}

Euripides Hatzikraniotis and Konstantinos M. Paraskevopoulos

Department of Physics, Aristotle University of Thessaloniki, 54124 Thessaloniki, Greece

Christos D. Malliakas

Department of Chemistry, Michigan State University, East Lansing, Michigan 48824

Jeff S. Dyck ${ }^{\text {a) }}$ and Ctirad Uher

Department of Physics, University of Michigan, Ann Arbor, Michigan 48109

Mercouri G. Kanatzidis ${ }^{\text {b) }}$

Department of Chemistry, Michigan State University, East Lansing, Michigan 48824

(Received 24 May 2006; accepted 24 August 2006; published online 19 December 2006)

\begin{abstract}
The charge transport properties of the low-dimensional thermoelectric materials $\mathrm{K}_{2} \mathrm{Bi}_{8-x} \mathrm{Sb}_{x} \mathrm{Se}_{13}$ $(0<x \leqslant 8)$ were studied as a function of temperature and composition. The Seebeck coefficient shows an evolution from $n$ - to $p$-type character with increasing incorporation of $\mathrm{Sb}$, and at the same time the temperature dependence of the electrical conductivity changes from that of a degenerate semiconductor to that of an intrinsic or compensated semiconductor. These changes, however, are not monotonic with composition due to the nonuniform substitution of $\mathrm{Sb}$ atoms at the Bi sites of the structure. Three separate composition regions can be assigned depending on $x$ each with different charge transport characters. Electronic transport in $\mathrm{K}_{2} \mathrm{Bi}_{8-x} \mathrm{Sb}_{x} \mathrm{Se}_{13}$ was analyzed on the basis of the classical semiconductor theory and discussed in the context of recent band calculations. The results suggest that the $\mathrm{K}_{2} \mathrm{Bi}_{8-x} \mathrm{Sb}_{x} \mathrm{Se}_{13}$ materials possess coexisting domains with semimetallic and semiconducting characters whose ratio is influenced by the value of $x$ and by local defects. The extent and relative distribution of these domains control the charge transport properties. Electron diffraction experiments performed on samples of $\mathrm{K}_{2} \mathrm{Bi}_{8-x} \mathrm{Sb}_{x} \mathrm{Se}_{13}$ with $x=1.6$ show evidence for such domains by indicating regions with long range ordering of $\mathrm{K}^{+} / \mathrm{Bi}^{3+}$ atoms and regions with increased disorder. The semiconducting behavior is enhanced with increasing $x$ (i.e., $\mathrm{Sb} / \mathrm{Bi}$ ratio) in the composition through a decrease of the semimetallic fraction. (C) 2006 American Institute of Physics. [DOI: 10.1063/1.2365718]
\end{abstract}

\section{INTRODUCTION}

Thermoelectric devices are based on heavily doped semiconductors and can be used for cooling or electricity generation directly from a heat source. A broad search is under way to identify materials with enhanced thermoelectric (TE) properties. Interest is now focused on materials with high figure of merit ${ }^{1}$ at high temperatures for direct energy conversion. Several classes of materials are currently under investigation, including complex chalcogenides, ${ }^{2,3}$ skutterudites, ${ }^{4,5}$ half-Heusler alloys, ${ }^{6}$ metal oxides ${ }^{7}$ and intermetallic clathrates. ${ }^{8} \beta-\mathrm{K}_{2} \mathrm{Bi}_{8} \mathrm{Se}_{13}$ is a chalcogenide with many attractive features that make it promising for thermoelectric applications. ${ }^{9-11}$ This compound has crystal and electronic structures ${ }^{12}$ that can lead to a high Seebeck coefficient ${ }^{13}$ and low thermal conductivity that arises from a large, low symmetry unit cell and weakly bound $\mathrm{K}^{+}$ions in cages ("phonon glass-electron crystal" conjecture). ${ }^{14} \beta$ $-\mathrm{K}_{2} \mathrm{Bi}_{8} \mathrm{Se}_{13}$ has a low symmetry monoclinic structure ${ }^{10}$ that

\footnotetext{
${ }^{a}$ Present address: Department of Physics, John Carroll University, University Heights, $\mathrm{OH}$.

${ }^{b)}$ Electronic mail: m-kanatzidis@ northwestern.edu
}

includes two different interconnected types of $\mathrm{Bi} / \mathrm{Se}$ building blocks [the so-called $\mathrm{NaCl}^{(100)}$ and $\mathrm{NaCl}^{(111)}$ types] and $\mathrm{K}^{+}$ atoms in tunnels. The two different $\mathrm{Bi} / \mathrm{Se}$ blocks are connected to each other at special mixed-occupancy K/Bi sites that seem to be crucial in defining the electronic structure near the Fermi level and consequently govern the electronic properties. This is supported by the results of $a b$ initio density functional band structure calculations on this compound. ${ }^{12}$ Its highly anisotropic structure results in needlelike morphology along the $b$ crystallographic axis.

Charge transport and thermal conductivity measurements on doped $\beta-\mathrm{K}_{2} \mathrm{Bi}_{8} \mathrm{Se}_{13}$ showed very low thermal conductivity $(\sim 1.3 \mathrm{~W} / \mathrm{m} \mathrm{K})$ and relatively high power factor $\left(S^{2} \sigma\right.$ $\left.\sim 12 \mu \mathrm{W} / \mathrm{cm} \mathrm{K}^{2}\right) .{ }^{10}$ The Seebeck coefficient is negative, indicating an $n$-type character, while its temperature dependence as well as that of electrical conductivity suggests a highly degenerate semiconductor. The charge transport properties have also been studied under pressure, where a significant increase in the power factor as well as a peak in the Seebeck coefficient was observed, suggesting electronic topological transition upon compression. ${ }^{15}$

In general, the formation of solid solutions is a common 
strategy to minimize the thermal conductivity and improve the thermoelectric performance of materials. Solid solutions based on the $\beta-\mathrm{K}_{2} \mathrm{Bi}_{8} \mathrm{Se}_{13}$ structure can be achieved via substitution at the heavy metal sites (i.e., $\mathrm{K}_{2} \mathrm{Bi}_{8-x} \mathrm{Sb}_{x} \mathrm{Se}_{13}$ ), ${ }^{16}$ the chalcogenide sites (i.e., $\mathrm{K}_{2} \mathrm{Bi}_{8} \mathrm{Se}_{13-x} \mathrm{~S}_{x}$ ), ${ }^{17}$ as well as at the alkali metal sites (i.e., $\mathrm{K}_{2-x} \mathrm{Rb}_{x} \mathrm{Bi}_{8} \mathrm{Se}_{13}$ ). ${ }^{18}$ Crystallographic studies show that partial substitution of $\mathrm{Bi} / \mathrm{Sb}$ (Ref. 19) as well as $\mathrm{K} / \mathrm{Rb}$ (Ref. 18) results in a nonuniform distribution of these atoms in the structure because of specific lattice site preferences of the substituting atoms. In contrast, $\mathrm{Se} / \mathrm{S}$ distribution ${ }^{17}$ was found to be more uniform throughout the structure, suggesting homogenous solid solutions in this case. The $\mathrm{Bi} / \mathrm{Sb}$ partial substitution decreases the lattice thermal conductivity ${ }^{20}$ of the compounds due to the mass and strain fluctuations introduced in the lattice.

In a previous work, ${ }^{16}$ the charge transport properties of selected members of the $\mathrm{K}_{2} \mathrm{Bi}_{8-x} \mathrm{Sb}_{x} \mathrm{Se}_{13}$ series displayed strong anisotropy and indicated the importance of growing highly oriented ingots. Moreover, compounds with low $\mathrm{Sb}$ content showed a degenerate semiconductor behavior with very high free carrier concentration $\sim 10^{20} \mathrm{~cm}^{-3}$. Investigations in the Bi rich region have shown that samples prepared with Se excess have better thermoelectric properties due to the higher electrical conductivity. ${ }^{16}$

In the present work, we examine the charge transport properties of $\mathrm{K}_{2} \mathrm{Bi}_{8-x} \mathrm{Sb}_{x} \mathrm{Se}_{13}$ prepared with $\mathrm{Se}$ excess in more detail and over a greater range of $x$. The aim was to understand the origin of the observed transport behavior (e.g., high free carrier concentration) and its characteristics. The charge transport properties have been interpreted in the light of recent band structure calculations on $\mathrm{K}_{2} \mathrm{Bi}_{8} \mathrm{Se}_{13}$ and in reference to the effect and extent of disorder between the $\mathrm{K} / \mathrm{Bi}$ metal atoms. The calculations indicated that, depending on how the $\mathrm{K}$ and $\mathrm{Bi}$ atoms are arranged in the mixed occupancy lattice sites, an energy gap may or may not appear at the Fermi level. Therefore, there exist two extreme variants of the band structure from a semimetal (band overlap) to semiconductor (band gap) based on the K/Bi arrangements.

To explain the charge transport properties we suggest a hybrid model involving both variants. Namely, a detailed analysis of the carrier concentration as well as of the Seebeck coefficient shows that the properties can be understood with a finite-gap semiconductor model that contains a significant concentration of shallow donors. The donor states likely originate from defects created by local disordering between $\mathrm{K}$ and $M(\mathrm{Bi}$ and $\mathrm{Sb})$. We present experimental evidence, through electron beam diffraction, of a long range order of $\mathrm{K} / \mathrm{Bi}$ atoms mixed with variable local atomic disorder. Furthermore, our results show that the higher the Sb concentration in $\mathrm{K}_{2} \mathrm{Bi}_{8-x} \mathrm{Sb}_{x} \mathrm{Se}_{13}$ is, the more pronounced the semiconducting behavior is as manifested by an increase of the band gap. The Seebeck coefficient of the compounds with $x$ $<5.6$ is negative at all temperatures. When $x \geqslant 5.6$, the compounds show a crossover from $n$-type to $p$ type with the decreasing temperature. The implications of this unique behavior on achieving a high thermoelectric figure of merit are discussed.

\section{EXPERIMENT}

\section{A. Sample preparation}

$\mathrm{K}_{2} \mathrm{Bi}_{8-x} \mathrm{Sb}_{x} \mathrm{Se}_{13}$ samples were prepared with $0.2 \mathrm{wt} \% \mathrm{Se}$ excess by mixing potassium metal, bismuth, antimony, and selenium which were loaded into a silica tube. For example, a $\mathrm{K}_{2} \mathrm{Bi}_{8-x} \mathrm{Sb}_{x} \mathrm{Se}_{13}(x=1.6)$ with $0.2 \%$ Se excess was prepared by mixing $0.271 \mathrm{~g} \mathrm{~K}(6.9 \mathrm{mmol}), 5.086 \mathrm{~g} \mathrm{Bi}(24.3 \mathrm{mmol})$, $0.741 \mathrm{~g} \mathrm{Sb}(6.1 \mathrm{mmol})$, and $3.911 \mathrm{~g} \mathrm{Se}(49.5 \mathrm{mmol})$. All manipulations were carried out under a dry nitrogen atmosphere in a Vacuum Atmospheres Dri-Lab glove box. The charged silica tube was flame-sealed at a residual pressure of $<10^{-4}$ Torr and heated to $850{ }^{\circ} \mathrm{C}$ over $12 \mathrm{~h}$ and kept there for $1 \mathrm{~h}$, followed by cooling to $50{ }^{\circ} \mathrm{C}$ at $-15^{\circ} \mathrm{C} / \mathrm{h}$. Highly oriented ingots with needlelike morphology were grown with a modified Bridgman technique. ${ }^{21}$ The purity of phases for the solid solutions was confirmed by comparison of $\mathrm{x}$-ray powder diffraction patterns to the calculated ones using CERIUS $^{2}$ software. ${ }^{22}$

\section{B. Transmission electron microscopy}

Selected area electron diffraction (SAED) measurements were performed on single crystals. The sample was mounted on a copper slotted grid and ion milled. Transmission electron microscopy (TEM) was performed on a JEOL JEM2200FS electron microscope.

\section{Charge transport measurements}

Transport property measurements were performed in a cryostat equipped with a radiation shield from 4 to $300 \mathrm{~K}$. Electrical conductivity, Hall effect, and thermopower measurements were carried on the same sample. The sample's typical size was $3 \times 3 \times 5 \mathrm{~mm}^{3}$. Electrical conductivity measurements were performed using a steady-state four-probe technique. The Hall effect was measured with the aid of a Linear Research bridge operated at $16 \mathrm{~Hz}$ excitation in a cryostat equipped with a magnet capable of fields up to 5.5 T. The Seebeck coefficient was determined using a longitudinal steady-state method over the temperature range of 4-300 K. The samples were attached (using either a low melting point solder or silver-loaded epoxy) to the cold tip of the cryostat with a thin-film resistor on the other (top) end that served as a heater. The temperature difference across the sample was measured using a differential chromelconstantan thermocouple and the Seebeck voltage was measured with thin copper wires, the thermopower of which was calibrated against a high- $T_{c}$ superconductor up to $134 \mathrm{~K}$, and against $\mathrm{Pb}$ at higher temperatures. ${ }^{23}$ Thermopower measurements were also extended to higher temperatures (300-700 K) with a programmable Seebeck controller SB100 from MMR Technologies, Inc. using constantan wire as reference. An electric current and heat gradient were applied along the needle direction (crystallographic $b$ axis).

\section{RESULTS AND DISCUSSION}

Charge transport measurements were carried out on the $\mathrm{K}_{2} \mathrm{Bi}_{8-x} \mathrm{Sb}_{x} \mathrm{Se}_{13}$ series for the compositions with $x=0.8,1.6$, $2.4,4.0,5.6$, and 8.0. The main goals were to understand the 
TABLE I. Room temperature values of the Seebeck coefficient $(S)$ and electrical conductivity $(\sigma)$ of $\mathrm{K}_{2} \mathrm{Bi}_{8-x} \mathrm{Sb}_{x} \mathrm{Se}_{13}$ series.

\begin{tabular}{ccc}
\hline \hline$x$ & $S(\mu \mathrm{V} / \mathrm{K})$ & $\sigma(\mathrm{S} / \mathrm{cm})$ \\
\hline 0.8 & -92 & 495 \\
1.6 & -74 & 746 \\
2.4 & -98 & 382 \\
4.0 & -161 & 56 \\
5.6 & -366 & 51 \\
8 & +730 & 0.01 \\
\hline \hline
\end{tabular}

underlying physics of charge transport in these complex materials. Of particular interest was the effect of Sb incorporation. The room temperature values of the Seebeck coefficient and electrical conductivity are given in Table I. Typical values for the electrical conductivity and Seebeck coefficient as a function of temperature are presented in Fig. 1. On the Bi-rich side of the composition both the conductivity and Seebeck coefficient appear typical for heavily doped semiconductors; a nearly monotonic decrease in electrical conductivity and a corresponding increase in absolute value of the Seebeck coefficient are observed. The mid $x$-range member $(x=4.0)$ shows a substantially lower conductivity, whereas the Seebeck coefficient has the same trend with the Bi-rich members, though higher in absolute values. It is clear that on the Bi-rich side of the composition, when the $\mathrm{Sb}$ content is increased the free carrier concentration is reduced.
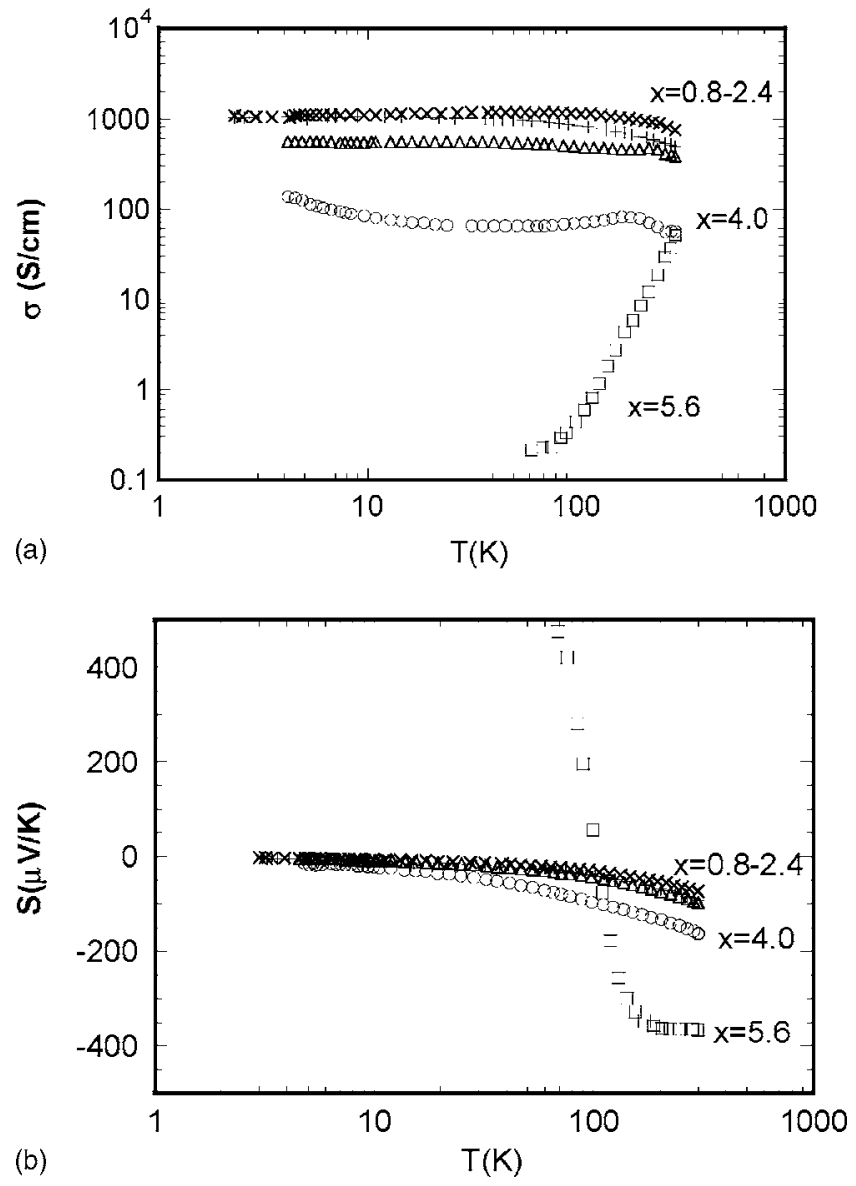

FIG. 1. (a) Electrical conductivity and (b) Seebeck coefficient for selected members of $\beta-\mathrm{K}_{2} \mathrm{Bi}_{8-x} \mathrm{Sb}_{x} \mathrm{Se}_{13}$ series in the temperature range of $4-300 \mathrm{~K}$.
When the $\mathrm{Sb}$ content is further increased, the material changes behavior completely. At $x=5.6$, the description is totally transformed from a heavily doped system into a typical semiconductor with thermally activated electrical conductivity. There is a change in the sign of the Seebeck coefficient to positive which with rising temperature changes to negative indicating that the Fermi level is close to the middle of the gap. This is consistent with the temperature dependence of conductivity (see below).

\section{A. Structure and charge transport}

The three-dimensional structure of $\beta-\mathrm{K}_{2} \mathrm{Bi}_{8} \mathrm{Se}_{13}$ includes two different interconnected types of $\mathrm{Bi} / \mathrm{Se}$ rod-shaped building blocks and $\mathrm{K}^{+}$atoms in tunnels, see Fig. 2. The so-called $\mathrm{NaCl}^{(100)}$-type and $\mathrm{NaCl}^{(111)}$-type blocks are structural fragments, interconnected to form a steplike structure along $a$ axis. $\mathrm{NaCl}^{(111)}$ type blocks are bridged to $\mathrm{NaCl}^{(100)}$-type $\mathrm{Bi} / \mathrm{Se}$ building blocks through sharing the $M(8)$ atom. The blocks are also connected at $M(9)$ metal sites with an $\mathrm{M}-\mathrm{Se}$ bond. $\mathrm{In}_{2} \mathrm{Bi}_{8} \mathrm{Se}_{13}$, the $M(8)$ and $M(9)$ are specially high ( 8 or 9 ) coordination sites that are mixedly occupied by $\mathrm{K}$ and $\mathrm{Bi}$ atoms.

Figure 3 shows the variation of free carrier concentration with temperature based on Hall Effect measurements for the Bi-rich $(x=0.8)$ member prepared with Se excess as well as data from Ref. 16 for the pure member (i.e., prepared without Se excess). Both sets of data exhibit a similar temperature behavior. The Hall coefficient is negative in value and suggests an $n$-type material that is in agreement with the Seebeck coefficient measurements. The Hall coefficient values are low at low temperature $\left[R_{\mathrm{Hall}}(5 \mathrm{~K})=-0.0564 \mathrm{~cm}^{3} / \mathrm{C}\right]$ and the corresponding carrier concentration $\left[n=1 /\left(R_{\text {Hall }} e\right)\right]$ is high, on the order of $10^{20} \mathrm{~cm}^{-3}$. High carrier concentrations at low temperatures are routinely observed in the Bi-rich members of the $\mathrm{K}_{2} \mathrm{Bi}_{8-x} \mathrm{Sb}_{x} \mathrm{Se}_{13}$ family. ${ }^{16}$

For comparison the temperature dependence of the free carrier concentration in $\mathrm{Bi}_{2} \mathrm{Se}_{3}$ samples is also included in Fig. 3. The difference between the two compounds is clear. The transport properties in $\mathrm{Bi}_{2} \mathrm{Se}_{3}$ can be understood within the framework of a narrow gap semiconductor but, as we will show below, this is not the case for the $\mathrm{K}_{2} \mathrm{Bi}_{8-x} \mathrm{Sb}_{x} \mathrm{Se}_{13}$ series. In $\mathrm{Bi}_{2} \mathrm{Se}_{3}$ (lower set of data in Fig. 3) the free carrier concentration ${ }^{24}$ is typically $\sim 10^{19} \mathrm{~cm}^{-3}$. Due to the small value of $E_{g}(0.30 \mathrm{eV})$, the transport properties are better described with a Kane model. ${ }^{25,26}$ The origin of the free carriers in $\mathrm{Bi}_{2} \mathrm{Se}_{3}$ is usually attributed to $\mathrm{Se}$ vacancies although antisite defects could also contribute. ${ }^{27} \mathrm{~A}$ higher free carrier concentration in $\mathrm{Bi}_{2} \mathrm{Se}_{3}$ can be achieved either by doping ${ }^{26,28}$ or with treating the compound with hydrazine. ${ }^{29,30}$ Even at very high carrier concentrations $\left(>10^{20} \mathrm{~cm}^{-3}\right)$, the transport properties in $\mathrm{Bi}_{2} \mathrm{Se}_{3}$ are consistent with a heavily doped $n$-type semiconductor transport with a Kane-type description of the conduction band. ${ }^{26,30}$

The carrier density in $\mathrm{K}_{2} \mathrm{Bi}_{8-x} \mathrm{Sb}_{x} \mathrm{Se}_{13}(x=0.8)$ increases slightly from the already high electron count of 1.1 $\times 10^{20} \mathrm{~cm}^{-3}$ measured at $5 \mathrm{~K}$ to $1.7 \times 10^{20} \mathrm{~cm}^{-3}$ at room temperature, which is by a factor of 10 higher than in pristine $\mathrm{Bi}_{2} \mathrm{Se}_{3}$. The origin of high free carrier concentration in 


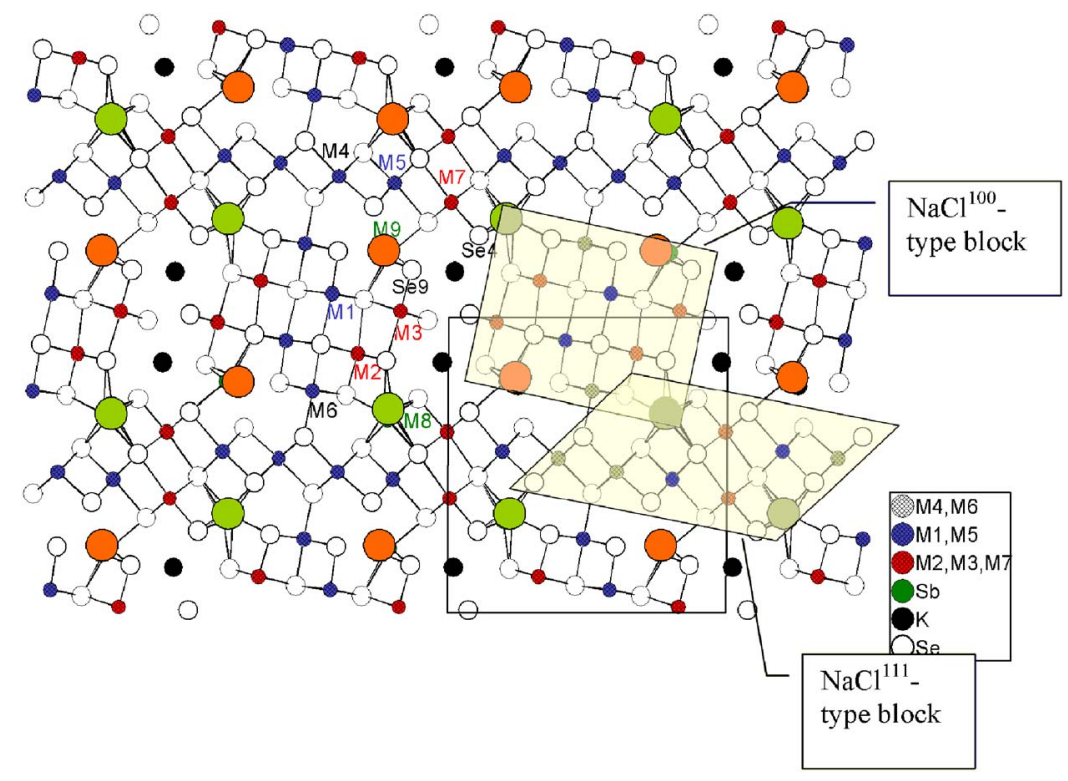

FIG. 2. (Color online) The crystal structure type of the $\mathrm{K}_{2} \mathrm{Bi}_{8-x} \mathrm{Sb}_{x} \mathrm{Se}_{13}$ series with atom labeling, viewed down the $b$ axis. $M(8)$ and $M(9)$ are the sites that connect the $\mathrm{NaCl}^{(111)}$ - and $\mathrm{NaCl}^{(100)}$-type blocks.

$\mathrm{K}_{2} \mathrm{Bi}_{8-x} \mathrm{Sb}_{x} \mathrm{Se}_{13}$ compounds is different from that in $\mathrm{Bi}_{2} \mathrm{Se}_{3}$ and will be discussed in detail below. Furthermore, $\mathrm{K}_{2} \mathrm{Bi}_{8-x} \mathrm{Sb}_{x} \mathrm{Se}_{13}$ compounds exhibit a much higher anisotropy, ${ }^{8}$ along and across the macroscopically developed needles, and electron transport would more resemble a quasione-dimensional system whereas $\mathrm{Bi}_{2} \mathrm{Se}_{3}$ is clearly twodimensional.

\section{Basic model for the electron transport}

In a typical semiconductor the electron carrier concentration $(n)$ in the parabolic approximation of the conduction band is given by ${ }^{31}$

$$
n=N_{C} F_{3 / 2}(\eta)=\frac{\left(2 m_{N} k T\right)^{3 / 2}}{3 \pi^{2} \hbar^{3}} F_{3 / 2}(\eta)
$$

where $m_{N}$ is the effective mass of electrons, $\eta$ is the reduced Fermi level $\left(\eta=E_{F} / k T\right), N_{C}$ is the effective density of states in the conduction band, and $F_{3 / 2}(\eta)$ is the Fermi integral

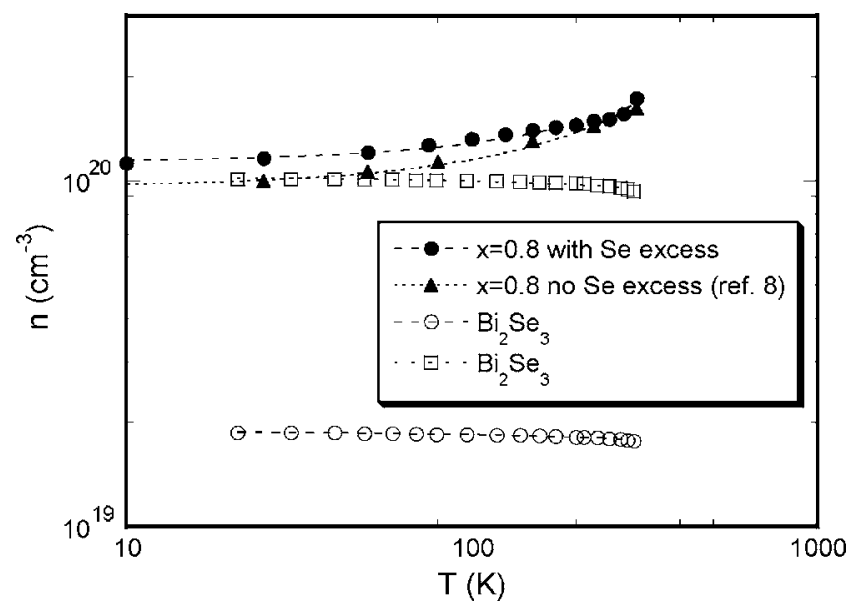

FIG. 3. Temperature dependence of the free carrier concentration for $x$ $=0.8$ of the $\mathrm{K}_{2} \mathrm{Bi}_{8-x} \mathrm{Sb}_{x} \mathrm{Se}_{13}$ series prepared with $\mathrm{Se}$ excess, in comparison with the same member $x=0.8$ without Se excess (from Ref. 8). Also included are data for $\mathrm{Bi}_{2} \mathrm{Se}_{3}$ : with two different carrier concentrations (data for $\mathrm{Bi}_{2} \mathrm{Se}_{3}$ were extracted from Ref. 19). which, in the general case with an index $r$, is given by

$$
F_{r}(\eta)=\int_{0}^{+\infty}\left[-\frac{\partial f(\eta)}{\partial \varepsilon}\right] \varepsilon^{r} d \varepsilon
$$

where $f$ represents the Fermi distribution function and $\varepsilon$ is the reduced energy. For arbitrary degeneracy of the electron gas, Eq. (1) does not have an analytical solution but, if $m_{N}$ is known, it may be solved numerically, resulting in the evaluation of the temperature dependence of the Fermi level. Similar to the parabolic approximation, in the Kane description for the conduction band, the free carrier concentration is given as

$$
n=\frac{\left(2 m_{N} k T\right)^{3 / 2}}{3 \pi^{2} \hbar^{3}} I_{3 / 2,0}^{0}(\eta, \beta)
$$

where

$$
I_{m, k}^{n}(\eta, \beta)=\int_{0}^{\infty}\left[-\frac{\partial f(\eta)}{\partial \varepsilon}\right] \varepsilon^{n} \frac{\left(\varepsilon+\beta \varepsilon^{2}\right)^{m}}{(1+2 \beta \varepsilon)^{k}} d \varepsilon \quad \text { and } \beta=\frac{k T}{E_{g}} .
$$

The nonparabolic Kane-type approximation becomes identical to the parabolic one when $\beta=0$. As it follows from Eq. (3), the effect of nonparabolicity is less profound at low temperatures as the small $k T$ term leads to $\beta \sim 0$. Furthermore, these effects should be more pronounced in $\mathrm{Bi}_{2} \mathrm{Se}_{3}$ than in $\mathrm{K}_{2} \mathrm{Bi}_{8} \mathrm{Se}_{13}$, since the energy gap $\left(E_{g}\right)$ is larger by a factor of $\sim 2$ in the later case. The effect of nonparabolicity is expected to be further reduced from the Bi-rich to the Sb-rich members, as the band gap widens.

The Fermi level is governed by the electroneutrality condition, which is the expression of the distribution of electrons and holes in the conduction/valence bands and in the donor/ acceptor states. In the case of a single donor level, the neutrality condition is

$$
n=p+\frac{N_{D}}{1+2 \exp \left(\varepsilon_{D}+\eta\right)},
$$

where $p$ is the hole concentration in the valence band, and the second term on the right-hand side refers to the donor 
level with concentration $N_{D}$ located at an energy $E_{D}\left(\varepsilon_{D}\right.$ $\left.=E_{D} / k T\right)$ below the bottom of the conduction band. If the donor level parameters $\left(N_{D}\right.$ and $\left.E_{D}\right)$ are known, Eq. (4) can be applied directly. Alternatively, $N_{D}$ and $\varepsilon_{D}$ can be evaluated as free parameters in a nonlinear fit of the experimental data for the carrier density $n(T)$, provided that $m_{N}, m_{P}$, and $E_{g}$ are known.

For a medium gap semiconductor with moderate doping, the free carrier concentration is almost constant at low temperatures (impurity saturation regime) and would rise at higher temperatures due to intrinsic transport. In a given temperature range, from low temperatures to room temperature, the overall variation of the free carrier concentration $(\Delta n)$ depends on the nature of the bands and the band gap. As the impurity concentration $N_{D}$ increases, the overall variation $(\Delta n / n)$ decreases; in heavily doped semiconductors, the free carrier concentration is almost temperature insensitive from $10 \mathrm{~K}$ to $300 \mathrm{~K}$. This qualitatively agrees with the observed temperature dependence of free carriers in $\mathrm{Bi}$ rich members (Fig. 3). As a result of this qualitative agreement, we should expect a high value of impurity concentration $\left(N_{D} \sim 10^{20} \mathrm{~cm}^{-3}\right)$.

\section{Atomic order/disorder in $\mathrm{K}_{2} \mathrm{Bi}_{8} \mathrm{Se}_{13}$}

Because of the mixed occupancy of the $M / \mathrm{K}$ sites $[M(8)$ and $M(9)]$ recent band structure calculations on $\mathrm{K}_{2} \mathrm{Bi}_{8} \mathrm{Se}_{13}$ dealt with the following two extreme cases of ordered arrangements: ${ }^{12}$ In case 1 there are two different configurations with extreme occupancy of the $M(8)$ and $M(9)$ (i.e., $\mathrm{K} 1 / \mathrm{Bi} 9$ and $\mathrm{K} 3 / \mathrm{Bi} 8$ ) sites such that each exclusively contained either $\mathrm{K}$ or $\mathrm{Bi}$ atoms (configurations $\mathrm{I}$ and II, respectively). ${ }^{32}$ In case 2 the $\mathrm{K}$ and $\mathrm{Bi}$ atoms are ordered along the $b$ axis creating a $1 \times 2 \times 1$ supercell with a repeat distance of $\sim 8.4 \AA^{12,33}$

In case 1 , configuration I involves a $\mathrm{Bi}$ atom occupying the $M(8)$ (i.e., $\mathrm{K} 3 / \mathrm{Bi} 8$ ) site and a $\mathrm{K}$ atom located at the $M(9)$ (i.e., K1/Bi9) site. The opposite exclusive-type occupancy, i.e., a $\mathrm{Bi}$ atom at the $\mathrm{K} 1 / \mathrm{B} 9$ site and a $\mathrm{K}$ atom at the $\mathrm{K} 3 / \mathrm{B} 8$ site, is referred to as configuration II. Configuration I, both before and after the inclusion of spin-orbit interaction, yielded a semimetallic behavior with $E_{g}=0$ and several flatbands in the plane perpendicular to the needle axis. Configuration II after the inclusion of spin-orbit interaction also gave $E_{g}=0$ with a band structure similar to that of configuration I.

In case 2 , which involves a $1 \times 2 \times 1$ supercell with ordered $\mathrm{Bi}$ and $\mathrm{K}$ distributions the band structure calculations showed a semiconductor with $E_{g}=0.55 \mathrm{eV}$, a value close to the spectroscopically observed gap ${ }^{16}$ of $0.60 \mathrm{eV}$.

These results underscore the importance of the mode of occupation of the $\mathrm{Bi}$ and $\mathrm{K}$ atoms on the $M(8)$ and $M(9)$ sites. To understand the electrical properties of the $\mathrm{K}_{2} \mathrm{Bi}_{8-x} \mathrm{Sb}_{x} \mathrm{Se}_{13}$ series, it is essential to consider this occupation especially due to the fact that $\mathrm{Sb}$ incorporation in the lattice is nonuniform. In addition, it is also important to understand the $\mathrm{Sb}$ incorporation in the lattice which occurs in stages.
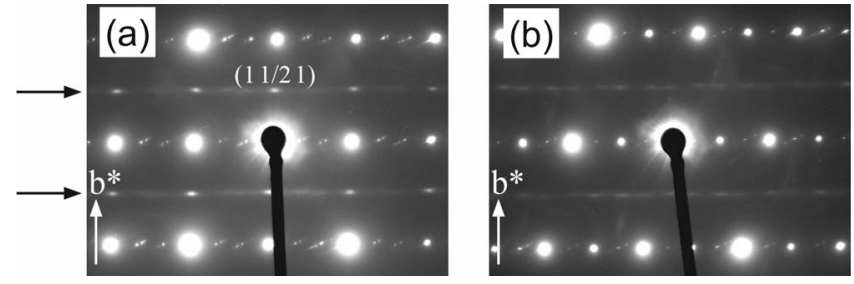

FIG. 4. Selected area electron diffraction (SAED) patterns of $\mathrm{K}_{2} \mathrm{Bi}_{64} \mathrm{Sb}_{16} \mathrm{Se}_{13}$. (a) Viewed down the $\left[\begin{array}{lll}1 & 2 & 0\end{array}\right]$ zone axis. (b) Section inclined with respect to the $\left[\begin{array}{lll}1 & 1 & 2\end{array}\right]$ zone. The black arrows indicate the positions of supercell Bragg reflections and diffuse intensity revealing the doubling of the $b$ axis. The diffuse intensity is more visible in (b).

\section{B. Sb incorporation in the lattice}

There are eight $\mathrm{Bi}$ atoms that occupy nine crystallographic sites in the structure $[M(1)-M(9)]$ out of which two are of mixed occupancy with $\mathrm{K}[M(8)$ and $M(9)]$. This can appear as a random occupancy in these two sites or as an ordered arrangement throughout the crystal. The former would be akin to case 1 and the latter to case 2 described above.

We have obtained direct experimental evidence for the existence and extent of the " $1 \times 2 \times 1$ superstructure" in Birich members of $\mathrm{K}_{2} \mathrm{Bi}_{8-x} \mathrm{Sb}_{x} \mathrm{Se}_{13}$ using TEM and selected area electron diffraction. Namely, as shown in the diffraction pattern of Fig. 4, for $x=1.6$ we observe Bragg spots that appear along the $b^{*}$ axis half way between the $(0 \mathrm{kl})$ lines of reflections. This indicates a clear doubling of the periodicity along the $b$ axis from 4.2 to $8.4 \AA$ consistent with case 2 . The arrows in Fig. 4(a) indicate the position of the extra diffracted intensity associated with long range ordering along the $b$ axis of $\mathrm{K}$ and $M$ atoms. In addition to the Bragg superlattice peaks we also observe diffuse scattering, see Fig. 4 (b), which clearly indicates the presence of disorder ${ }^{34}$ consistent with case 1 .

The substitution of $\mathrm{Bi}$ for $\mathrm{Sb}$ atoms does not form true solid solutions in this system. It is, in fact, preferential ${ }^{19}$ due to the large number of different metal sites in the structure. In the Bi-rich region, the cell volume significantly deviates ${ }^{16}$ from Vegard's law at compositions in the vicinity of $x$ $=1.5-2.0$. In the same compositional region, the energy gap shows an anomaly with a minimum at $x=1.5$ and this is also reflected in the higher electrical conductivity and lower Seebeck coefficient for this member [Fig. 5(a)].

The $M(8)$ and $M(9)$ sites that serve to join the $\mathrm{NaCl}^{(111)}$-type and the $\mathrm{NaCl}^{(100)}$-type blocks are the most receptive to $\mathrm{Sb}$ atoms and they are the first ones to be substituted at small $x$. For $x=2.97$, for example, 1.50 of $\mathrm{Sb}$ resides at $M(8)$ and $M(9)$ sites and the rest (1.47) Sb atoms are distributed in $M(1)-M(7)$. For $x>1.5$, Sb atoms tend to occupy $M(1), M(2), M(3), M(5)$, and $M(7)$ sites. In contrast, sites $M(4)$ and $M(6)$ accommodate $\mathrm{Sb}$ atoms only at high values of $x$. Note that the $\mathrm{NaCl}^{(100)}$-type $\mathrm{Bi} / \mathrm{Se}$ building blocks include heavy metal sites $M(1), M(2), M(3)$, and $M(6)$ and the $\mathrm{NaCl}^{(111)}$-type blocks include $M(4), M(5)$, and $M(7)$.

At the Sb-rich side of the composition the energy gap changes slope at $x \sim 6.0$ increasing faster for higher $x$ values. ${ }^{16}$ The electrical conductivity shows clear semicon- 

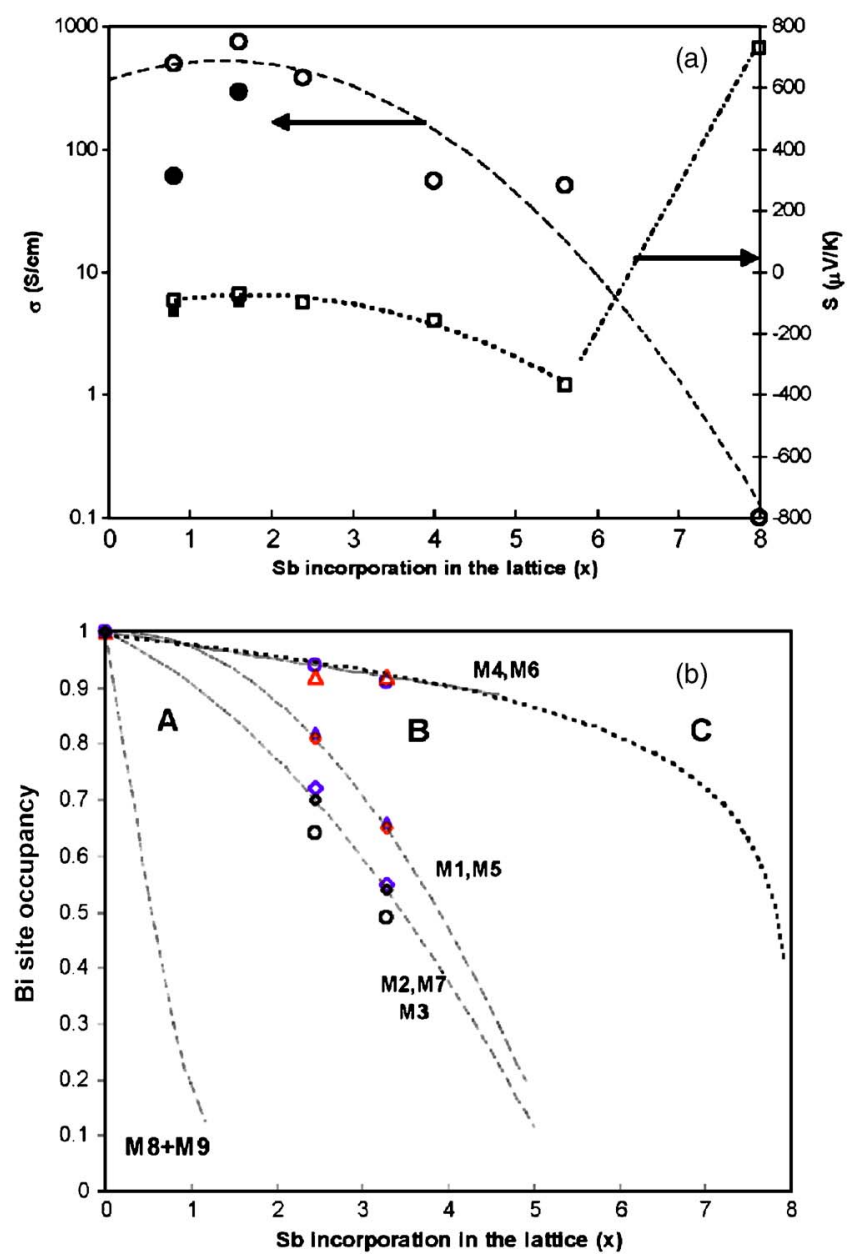

FIG. 5. (Color online) (a) Room temperature electrical conductivity and Seebeck coefficient with $\mathrm{Sb}$ incorporation. Filled circles and squares are data from Ref. 8 and correspond to samples prepared without Se excess. (b) Bi occupancies of each crystallographic site for specific $x$ value (i.e., degree of $\mathrm{Sb}$ incorporation). Obviously for $x=0$ the Bi occupancy is 1 and sites $M(8)$ and $M(9)$ contain no $\mathrm{Sb}$ atoms. Lines are guides to the eye. The three different composition regions are shown as $\mathrm{A}, \mathrm{B}$, and $\mathrm{C}$.

ducting behavior [Fig. 1(a)] and the Seebeck coefficient also shows a $p$-to- $n$ transition for the $x=5.6$ member [Fig. 1(b)].

The site preferences for $\mathrm{Bi} / \mathrm{Sb}$ substitution are drawn in Fig. 5(b) with Bi occupancy on each crystallographic site versus $\mathrm{Sb}$ incorporation in the lattice. Obviously, for $x=0$ the Bi occupancy in all $M(1)-M(7)$ sites is 1 . The dotted lines in Fig. 5(b) are simply quadratic extrapolations for the higher $\mathrm{Sb}$ content and mostly serve as a guide to the eye in order to group together sites with similar behavior.

For the purposes of this discussion we divided the $\mathrm{K}_{2} \mathrm{Bi}_{8-x} \mathrm{Sb}_{x} \mathrm{Se}_{13}$ family into the following three separate composition regions on the basis of the observed trends in $\mathrm{Sb} / \mathrm{Bi}$ substitution ${ }^{35}$ at different sites:

Region A is a Bi-rich region, where Sb preferentially occupies the sites $M(8)$ and $M(9)$. The boundary of this region cannot be precisely defined; it is estimated that it may extend up to $x=1.5$ or 1.7 since, for higher $x$ values, the $\mathrm{Bi}$ concentration on the $M(8)$ and $M(9)$ sites was found to be zero. $^{19}$

Region $\mathrm{B}$ is a midregion, where $M(8)$ and $M(9)$ are already mainly occupied by $\mathrm{Sb}$ and $\mathrm{K}$. A preferential $\mathrm{Bi} / \mathrm{Sb}$

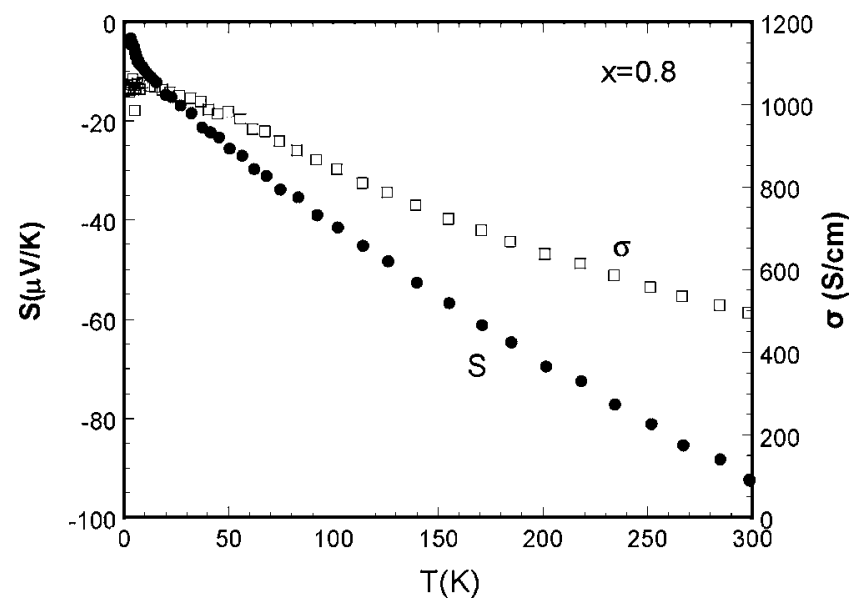

FIG. 6. Temperature dependence of the Seebeck coefficient and electrical conductivity $(4-300 \mathrm{~K})$ for the member $x=0.8$ of the $\mathrm{K}_{2} \mathrm{Bi}_{8-x} \mathrm{Sb}_{x} \mathrm{Se}_{13}$ series (region A).

substitution appears on sites $M(1), M(2), M(3), M(5)$, and $M(7)$. These sites have a slightly distorted octahedral coordination environment. ${ }^{10}$ Sites with less distorted octahedral coordination, i.e., $M(4)$ and $M(6)$, are still occupied by Bi. Sites $M(1)$ and $M(5)$, and $M(2), M(3)$, and $M(7)$ seem to group together. We estimate that $\mathrm{Sb}$ substitution in these sites to be complete at stoichiometries close to $x \sim 5.4-5.7$.

Region $\mathrm{C}$ is a $\mathrm{Sb}$-rich region, where $\mathrm{Sb}$ atoms begin to dominate the $M(4)$ and $M(6)$ sites. These sites quickly fill with $\mathrm{Sb}$ atoms at $x>5.5$.

\section{Analysis of charge transport properties}

Because the charge transport properties vary with the fraction of $\mathrm{Sb}$, the three composition regions will be discussed separately. The experimental data were analyzed based on the electron transport theory in the context of both the parabolic and nonparabolic band approximations as described above.

\section{Region A}

Seebeck coefficient and electrical conductivity data for the sample with $x=0.8$ at $4-300 \mathrm{~K}$ that belongs to this region are shown in Fig. 6. The Seebeck coefficient is negative and it tends towards zero as the temperature is decreased. The electrical conductivity shows a metallike character and Hall effect measurements indicate a high carrier concentration.

To fit the experimental charge transport data to a physical model we consider two cases depending on the nature of the energy gap. First is case 1, a zero-gap semiconductor followed by case 2, a finite-gap semiconductor $E_{g}>0$ (based on the $1 \times 2 \times 1$ supercell).

a. The case of zero-gap semiconductor: Case 1. The $\mathrm{K} / \mathrm{Bi}(\mathrm{Sb})$ occupancy distribution of case 1 gives $E_{g}=0$, and in the absence of impurities, the neutrality condition given by Eq. (4) becomes

$$
\left(m_{N}\right)^{3 / 2} F_{3 / 2}(\eta)=\left(m_{P}\right)^{3 / 2} F_{3 / 2}(-\eta) \text {. }
$$

As the $F_{r}(\eta)$ integrals are monotonic functions of $\eta$, the solution to Eq. (5), with respect to the reduced Fermi level, 
would be unique ( $\eta=\eta_{0}=$ const $)$ and independent of temperature and would, of course, depend only on the ratio $m_{N} / m_{P}$. The free carrier concentration would then have a $T^{3 / 2}$ dependence, due to the temperature dependence of the density of states $N_{C}$. The Seebeck coefficient in a parabolic or in Kane-type approximation is expressed as ${ }^{31}$

$$
S= \pm \frac{k}{e}\left[\frac{F_{r+2}(\eta)}{F_{r+1}(\eta)}-\eta\right] \quad \text { or } S= \pm \frac{k}{e}\left[\frac{I_{r+1,2}^{1}(\eta, \beta)}{I_{r+1,2}^{0}(\eta, \beta)}-\eta\right],
$$

where the index $r$ refers to the scattering mechanism. From the above equation the Seebeck coefficient would be expected to be temperature independent contrary to the experimental data (see Fig. 6).

In the general case where the impurities act as donors in a zero-gap semiconductor with $m_{P}>m_{N}$ (Ref. 36) there should be no bound electrons in the donor levels; therefore all donors will remain ionized even at $T=0$. The neutrality condition based on the Fermi approximation of the conduction band then becomes

$$
\frac{\left(2 m_{N} k T\right)^{3 / 2}}{3 \pi^{2} \hbar^{3}} F_{3 / 2}(\eta)=\frac{\left(2 m_{P} k T\right)^{3 / 2}}{3 \pi^{2} \hbar^{3}} F_{3 / 2}(-\eta)+N_{D} .
$$

The free carrier concentration should remain constant at low temperatures and equal to $N_{D}$, which is in agreement with the high free carrier concentration observed at low temperatures. As the temperature rises, all donors remain ionized, and the free carrier concentration increases monotonically due to the excitation of electrons from the valence to the conduction band. However, because $\eta$ is temperature dependent, according to Eq. (7), the rate of increase of free carriers is expected to be proportional to $T^{3 / 2}$, a dependence that is much steeper than the one observed. Therefore, the electron transport for the $x=0.8$ compound cannot be understood assuming a zero-gap semiconductor. We can also conclude from this analysis that the crystal structure of $\mathrm{K}_{2} \mathrm{Bi}_{8-x} \mathrm{Sb}_{x} \mathrm{Se}_{13}$ represented exclusively by case 1 is not sufficient to describe the full character of these materials. ${ }^{37}$ Consequently, we proceeded to examine case 2 in the same context.

b. The case of finite-gap semiconductor: Case 2. In case 2 we accept a finite energy gap $\left(E_{g}>0\right)$ for the system and consider standard semiconductor statistics, with the presence of impurities that act as electron donors [Eq. (4)]. The position of the Fermi level $E_{F}$ was calculated at different temperatures from Seebeck measurements using Eq. (6), and then the free carrier concentration was evaluated through Eq. (1). The density of states effective mass $\left(m_{N}\right)$ was set as a free parameter, so that the calculated free carrier concentration $n_{\text {calc }}$ matched the experimentally measured one at high temperatures. Namely, we considered two extreme cases of scattering, specifically carrier scattering by acoustic phonons $(r=0)$ and impurity scattering $(r=2)$, as well as two models describing the conduction band: the parabolic approximation and the nonparabolic Kane-type model.

The temperature variation of the Fermi level, as calculated on the basis of the parabolic $\left(E_{\mathrm{FF}}\right)$ and nonparabolic models $\left(E_{\mathrm{FK}}\right)$ for $r=0$, is given in Fig. 7(a). In both cases, the
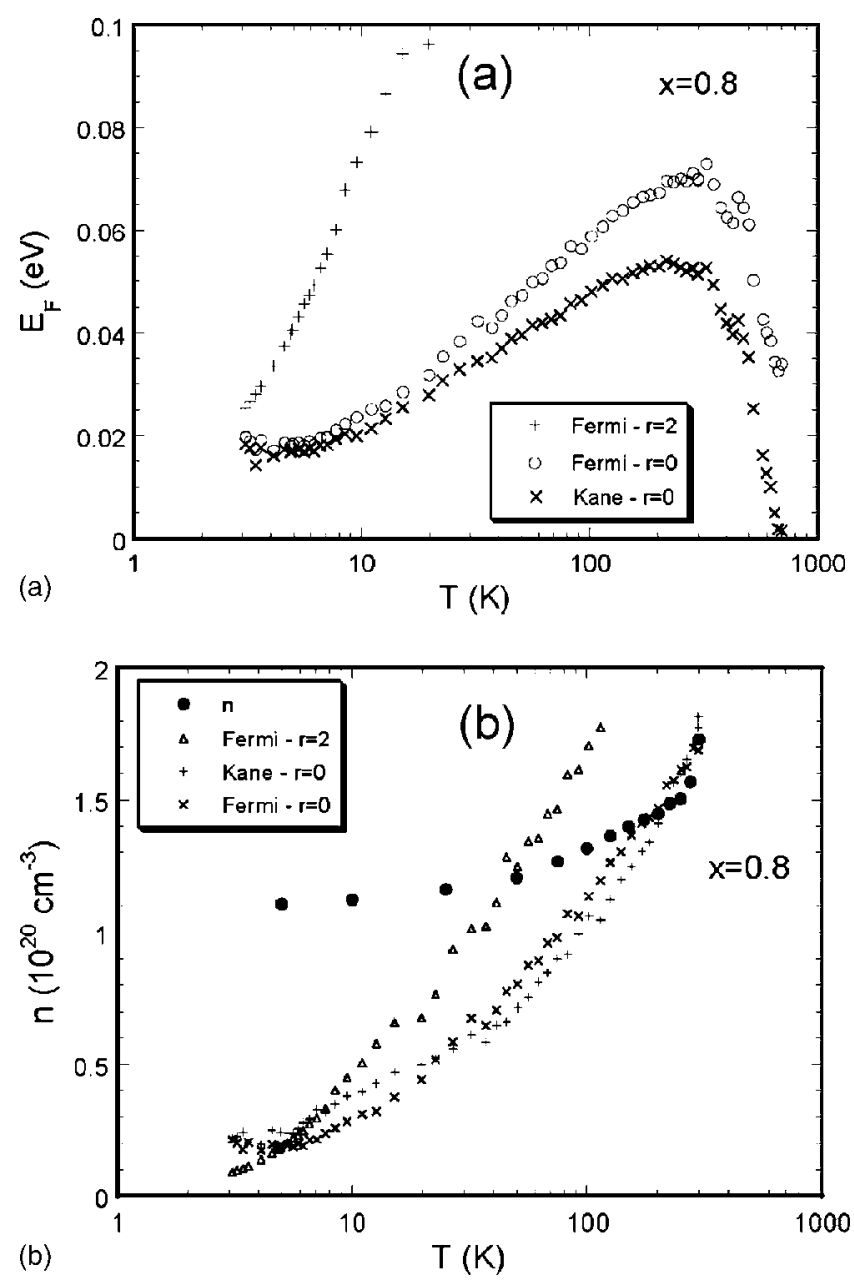

FIG. 7. (a) Temperature dependence of the Fermi level, as calculated on the basis of parabolic $\left(E_{\mathrm{FF}}\right)$ for $r=0$ and $r=2$ and nonparabolic band model $\left(E_{\mathrm{FK}}\right)$ for $r=0$ [Eq. (6)]. (b) Temperature dependence of the experimental free carrier concentration $\left(n=1 / R_{H} e\right)$ and calculated on the basis of parabolic and nonparabolic models. Full circles correspond to experimental data for member $x=0.8$.

Fermi level lies well inside the conduction band. As the temperature increases, the Fermi level also increases, reaching a maximum, and when the donor levels are depleted it begins to decrease. The temperature dependence is consistent with the semiconductor theory. The Fermi level has higher values for the parabolic approximation, and this accounts for the difference in the density of states in the two approximations. Despite this difference, both treatments give similar results for the free carrier concentration Fig. 7(b).

When we compare the calculated free carrier concentrations, $n_{F}$ and $n_{K}$, obtained for the parabolic and nonparabolic model, respectively, with the experimentally measured one $(n)$, we observe significant deviations especially at low temperatures. Values calculated based on either model span a much wider range than those observed experimentally. A significant deviation between the experimental and calculated data is also observed in the two extreme cases of scattering, $r=0$ (acoustic phonons) and $r=2$ (impurity scattering) [Fig. 7(b)]. We conclude from this analysis that a model consistent with case 2 alone is also inadequate in accounting for the experimental observations in any of the band approximations. 


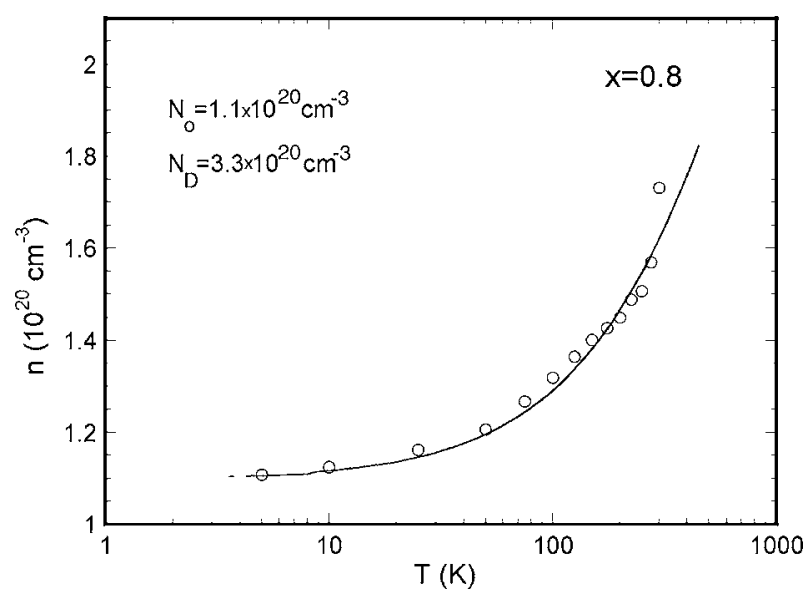

FIG. 8. Temperature dependence of the free carrier concentration for the member $x=0.8$ of the $\mathrm{K}_{2} \mathrm{Bi}_{8-x} \mathrm{Sb}_{x} \mathrm{Se}_{13}$ series (region $\mathrm{A}$ ). The solid line is calculated based on the neutrality condition and an additional constant term [Eq. (8)].

c. Extending the finite-gap model. As we mentioned above, according to the band structure calculations, the energy gap is very sensitive to how the $\mathrm{K} / \mathrm{Bi}(\mathrm{Sb})$ atoms are distributed in the $M(8)$ and $M(9)$ sites of mixed occupancy in case 1. Configurations I and II lead to a zero-gap semiconductor. In case 2 where the $\mathrm{K}$ and $\mathrm{Bi}$ atoms are regularly alternated in the $M(8)$ and $M(9)$ sites, the system becomes a semiconductor.

In neither case, however, can the electron transport be explained. Since in the real system, cases 1 and 2 seem to coexist a combined model needs to be introduced. Such a model could be created by starting from case $2\left(E_{g}>0\right)$ and introducing massive disorder midgap donor states originating from case 1 .

A spectacularly better agreement is obtained if an additional constant term $\left(N_{0}\right)$ is included in the neutrality condition. In reference to Eq. (4), the neutrality condition, with the addition of the constant term, is written as

$$
n=p+\frac{N_{D}}{1+2 \exp \left(\varepsilon_{D}+\eta\right)}+N_{0} .
$$

In the context of semiconductor statistics, the term $N_{0}$ would act as an additional donor level with no bound electron states; this donor level is ionized even at $T=0 \mathrm{~K}$. To simplify the application of the model, we considered an approximately parabolic conduction band with $m_{N}=m_{P}$, and $E_{D} \approx 0 \mathrm{eV}$. Both $N_{D}$ and $N_{0}$ were left as adjustable parameters. The best fit was obtained for $N_{D}=3.3 \times 10^{20} \mathrm{~cm}^{-3}$ and $N_{0}=1.1 \times 10^{20} \mathrm{~cm}^{-3}$, which agrees well with the measured carrier concentration at low temperatures, Fig. 8. The existence of these shallow donor levels is essential in order to explain the observed electron transport. Below we will discuss the nature and origin of these energy levels, which lie in the fine details of the atomic structural arrangement.

Configurations I and II occurring perhaps as islands inside the $1 \times 2 \times 1$ superstructure could create shallow energy levels close to the conduction band, i.e., $N_{0}$ sites that remain ionized even at very low temperatures. The relative ratio of these domains would depend on the growth conditions. In the $1 \times 2 \times 1$ superstructure, we have a sequence of $\mathrm{K}-\mathrm{Bi}-$ $\mathrm{K}-\mathrm{Bi}-\cdots \cdot$ occupancy at the $M(8) / M(9)$ sites. Local intermixing of $\mathrm{K}$ and $\mathrm{Bi}$ would lead to short sequences of $\cdots-\mathrm{K}-\mathrm{K}-$ $\mathrm{K}-\cdots-\mathrm{Bi}-\mathrm{Bi}-\mathrm{Bi}-\cdots$ which correspond to a structure close to configuration I and/or II.

The argument that shallow energy levels could be created close to the conduction band is supported by band orbital analysis in the case of configurations I and II which has shown that when $\mathrm{K}$ is at the $M(9)$ position, the $\mathrm{Se} 9$ atom has two $\mathrm{K}$ atom neighbors and its $p$ orbitals are destabilized and float to the Fermi energy giving rise to semimetallic behavior. ${ }^{12}$ Similarly, if $\mathrm{K}$ is at $M(8)$ position (configuration II) the $\mathrm{Se} 4$ atom (instead of $\mathrm{Se} 9$ ) is now in the neighborhood of two $\mathrm{K}$ atoms, and the $\mathrm{Se} 4 p$ orbitals get destabilized and float to the Fermi energy. Therefore, inclusions of domains of configuration I or II could generate massive midgap levels which would serve as shallow electron donors. These levels could account for the origin of the $N_{0}$ donor levels.

After the additional $N_{0}$ source of carriers is taken into account the Seebeck coefficient was recalculated, as the weighted average of the two contributions: the semiconducting one that originates from the $1 \times 2 \times 1$ supercell and the semimetallic contribution arising from configuration I or II inclusions. The Seebeck coefficient is then expressed as

$$
S=\frac{\sigma_{\mathrm{sm}} S_{\mathrm{sm}}+\sigma_{\mathrm{sc}} S_{\mathrm{sc}}}{\sigma_{\mathrm{sm}}+\sigma_{\mathrm{sc}}},
$$

where the subscripts sm and sc refer to the semimetallic and semiconducting contributions, respectively. In the simplest case, we assume that the carriers are scattered by the same mechanism, and Eq. (9) can be simplified and rewritten in terms of the free partial carrier concentrations as

$$
S n=n_{\mathrm{sm}} S_{\mathrm{sm}}+n_{\mathrm{sc}} S_{\mathrm{sc}},
$$

where $S$ are experimentally measured values for the Seebeck coefficient in a wide temperature range $(4-700 \mathrm{~K})$, and $n$ are based on experimentally measured data (see Fig. 6) that were fitted using Eq. (8). Based on Eq. (8), we first evaluated the Fermi level and subsequently the free carrier concentration $n_{\mathrm{sc}}$ [Eq. (1)] and the Seebeck coefficient [Eq. (6)]. In Eq. (10), $n_{\text {sm }}$ was taken equal to $N_{0}$, and $S_{\text {sm }}$ was finally calculated. As seen in Fig. 9, the measured Seebeck coefficient (combined low and high temperature data) for the $x=0.8$ compound is closer to the $S_{\mathrm{sm}}$ rather than to the $S_{\mathrm{sc}}$ description consistent with the fact that the system is degenerately doped by virtue of the shallow donor states.

Crystal growth conditions are expected to strongly affect the disorder in this system and consequently the charge transport in this region. Therefore, a gradual reduction of the disorder through proper optimization of the crystals is expected to reduce the shallow donor levels $N_{0}$. This in turn should reduce the sm contribution and potentially enhance the thermoelectric properties.

\section{Regions $B$ and $C$}

In region $\mathrm{B}$, the additional $\mathrm{Sb}$ atoms show a preferential substitution for the sites with distorted octahedral coordination environment which are proximal to the mixed sites $M(8)$ 


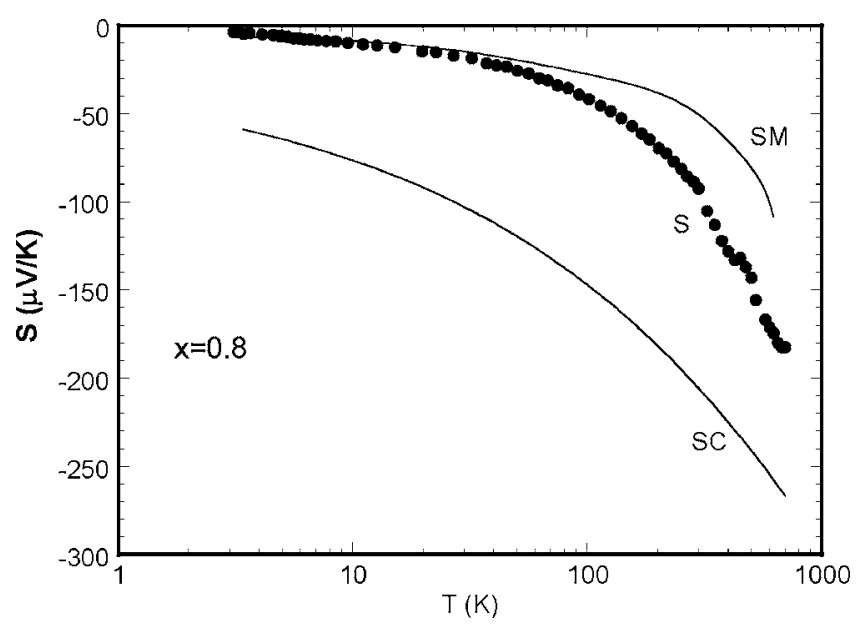

FIG. 9. Temperature dependence of the experimental and calculated Seebeck coefficient $(4-700 \mathrm{~K})$ based on both semiconducting (sc) and semimetallic (sm) contributions [Eq. (10)]. Full circles correspond to experimental data for member $x=0.8$ for temperatures up to $700 \mathrm{~K}$.

and $M(9)$. These are $M(2), M(3), M(7), M(1)$, and $M(5)$. The incorporation of $\mathrm{Sb}$ raises the energy of the conduction band resulting in a greater energy gap. This was confirmed experimentally in optical spectroscopic measurements. ${ }^{16}$

As the $\mathrm{Sb}$ content is increased $(x>1.6)$, the disorder at the $\mathrm{K} /(\mathrm{Bi}$ and $\mathrm{Sb})$ sites should tend to decrease. The $\mathrm{K}$ and $\mathrm{Bi}$ pair has a better ionic size match than the $\mathrm{K}$ and $\mathrm{Sb}$ pair and concequently the disorder at the mixed sites is, in fact, gradually eliminated as the composition changes towards the $\mathrm{Sb}$-rich end member. This is supported by detailed crystallographic studies on $\mathrm{K}_{2} \mathrm{Sb}_{8} \mathrm{Se}_{13}$, that show ordering of $\mathrm{K}$ and $\mathrm{Sb}$ occupancies giving a structure similar to the $1 \times 2 \times 1$ model of case 2. In this case, $\mathrm{Sb}$ and $\mathrm{K}$ are fully ordered and alternate in the connecting sites $M(8)$ and $M(9)$, doubling the crystallographic axis in that direction. ${ }^{38}$

The gradual elimination of the disorder at $M(8)$ and $M(9)$ is expected to remove the midgap levels (the source of $N_{0}$ ) and reveal the semiconducting character of $\mathrm{K}_{2} \mathrm{Bi}_{8-x} \mathrm{Sb}_{x} \mathrm{Se}_{13}$. This, however, does not yet happen at $x$ $=1.6$ and the large number of shallow donor levels persists or even grows. Indeed the $x=1.6$ compound (a "marginal" member at the boundary between regions A and B) shows a lower Seebeck coefficient than the one with $x=0.8$ suggesting a higher free carrier concentration [Figs. 6 and 10(a), respectively]. The Fermi level analysis based on Eq. (6) confirms this suggestion as it shows that the Fermi level lies at a higher energy for the $x=1.6$ compound than for the $x=0.8$ compound, Fig. 11. If we apply the same analysis of Eq. (8) in the $x=1.6$ compound, we obtain $N_{0}=1.65 \times 10^{20} \mathrm{~cm}^{-3}$.

Seebeck coefficient and electrical conductivity data for compounds with $x=2.4$, and 4.0, which lie well inside region B, are shown in Fig. 10. The Seebeck coefficient is negative for all samples in the temperature range of 4-300 K. The temperature dependence of the Seebeck coefficient and electrical conductivity exhibits similar characteristics as in the Bi-rich region $\mathrm{A}$, but becomes more pronounced at higher $x$ values. At $x=2.4$ and 4.0, the increase in the absolute value of the Seebeck coefficient (see also Table I) suggests the gradual elimination of the shallow donor levels, Fig. 10(a).
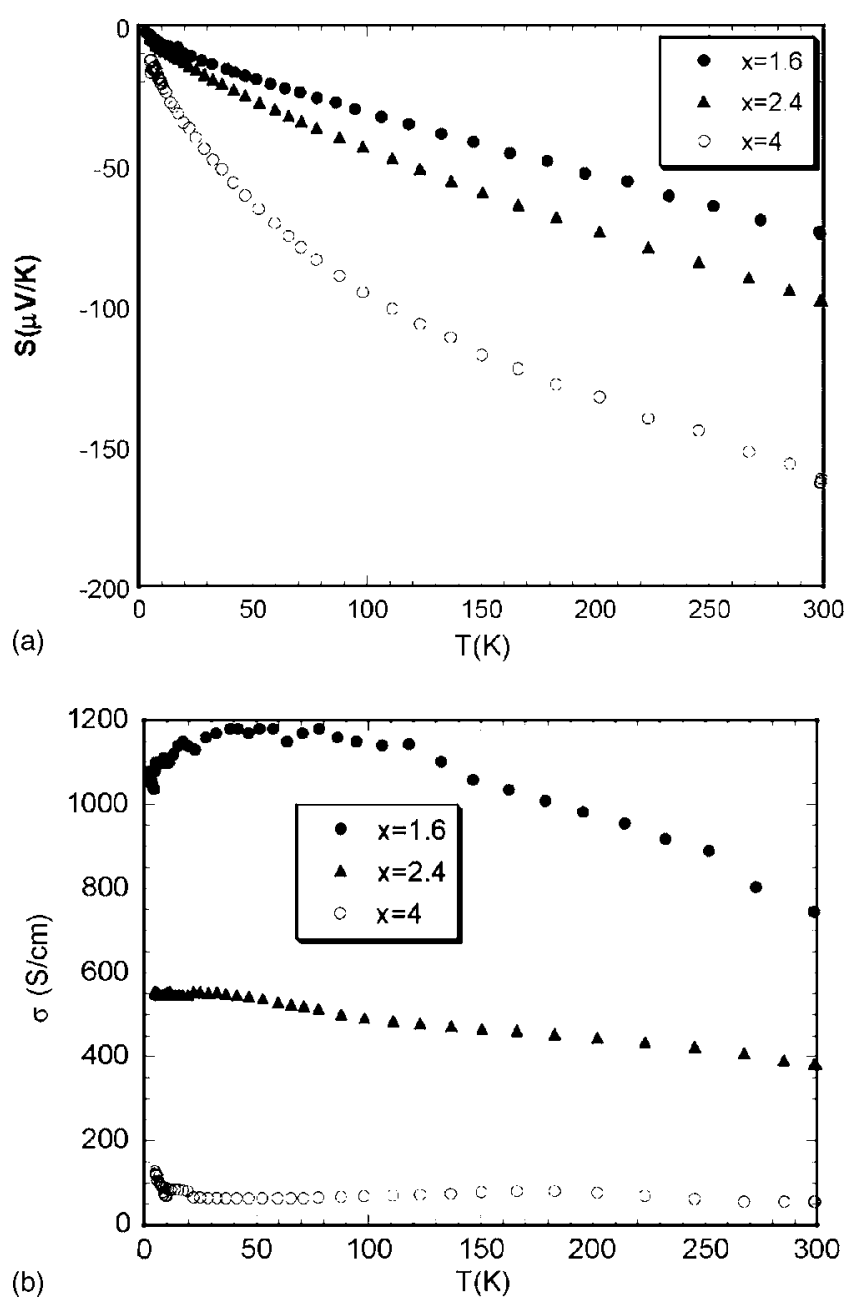

FIG. 10. Temperature dependence of (a) Seebeck coefficient and (b) electrical conductivity for the members $x=1.6,2.4$, and 4.0 of the $\mathrm{K}_{2} \mathrm{Bi}_{8-x} \mathrm{Sb}_{x} \mathrm{Se}_{13}$ series (region $\mathrm{B}$ ).

The Fermi level moves lower in energy at higher Sb concentrations, see Fig. 11 for $x=4.0$. The electrical conductivity also decreases and this is consistent with fewer $n$-type carriers [Fig. 10(b)].

In region $\mathrm{C}$ the additional $\mathrm{Sb}$ atoms are observed to invade the last heavy metal sites $[M(4)$ and $M(6)]$ in the crys-

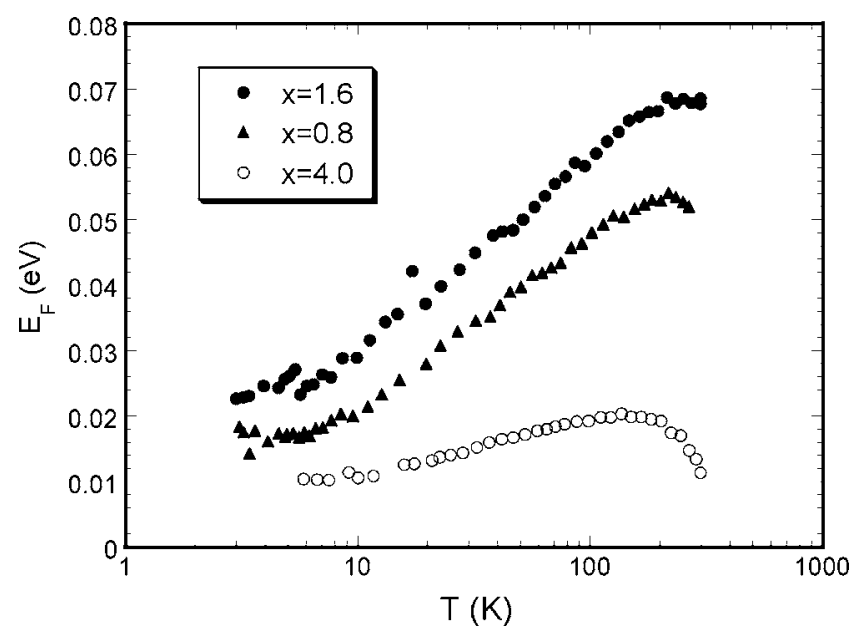

FIG. 11. Comparison of the Fermi level of $x=0.8$ (region A) and $x=1.6$ and 4.0 (region B) of the $\mathrm{K}_{2} \mathrm{Bi}_{8-x} \mathrm{Sb}_{x} \mathrm{Se}_{13}$ series. 


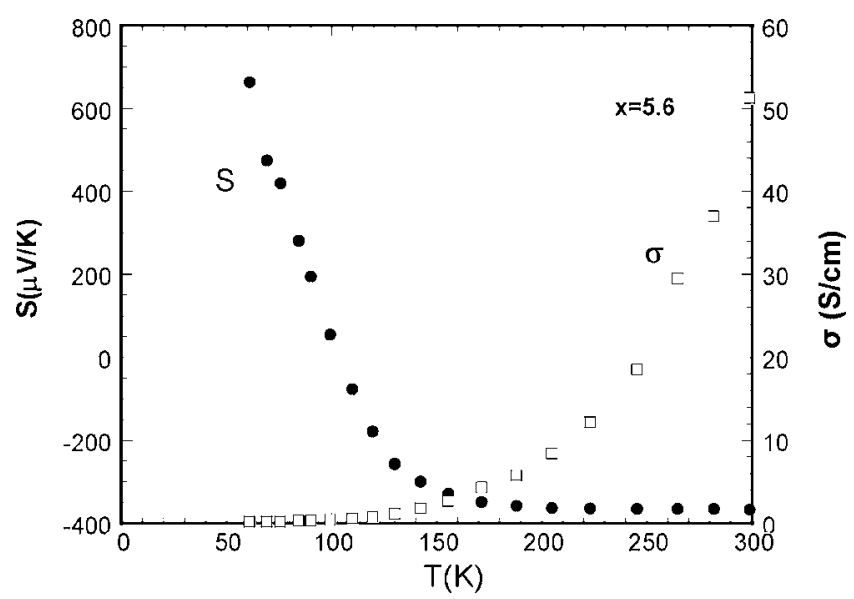

FIG. 12. Temperature dependence of the Seebeck coefficient and electrical conductivity for the member $x=5.6$ of the $\mathrm{K}_{2} \mathrm{Bi}_{8-x} \mathrm{Sb}_{x} \mathrm{Se}_{13}$ series (regions $\mathrm{B}$ and $\mathrm{C}$ ).

tal lattice. These compounds acquire a $p$-type character. The Seebeck coefficient of $\mathrm{K}_{2} \mathrm{Sb}_{8} \mathrm{Se}_{13}(x=8.0)$ is positive at $\sim$ $+730 \mu \mathrm{V} / \mathrm{K}$ at room temperature (see Table $\mathrm{I}$ ). The $x=8.0$ compound has a large $E_{g} \sim 0.8 \mathrm{eV}$ and the electrical conductivity is the lowest of all members $(0.01 \mathrm{~S} / \mathrm{cm}$ at room temperature).

The change from $n$ to $p$ character with the increasing $\mathrm{Sb}$ atom content occurs somewhere between regions $\mathrm{B}$ and $\mathrm{C}$. We studied the compound with $x=5.6$ which lies in region $\mathrm{C}$ yet close to the boundary with region B. The Seebeck coefficient is positive at low temperatures and at $>100 \mathrm{~K}$ it becomes negative, Fig. 12. This indicates a change in the predominant carrier from holes to electrons. At temperatures above $150 \mathrm{~K}$ the Seebeck coefficient appears as almost temperature independent at $\sim-370 \mu \mathrm{V} / \mathrm{K}$ up to room temperature. The electrical conductivity for the compound with $x$ $=5.6$ increases rapidly with rising temperature reaching $\sim 60 \mathrm{~S} / \mathrm{cm}$ at $300 \mathrm{~K}$. The thermally activated charge transport behavior is consistent with the observed transition of the Seebeck coefficient (Fig. 12). This suggest that the so-called shallow donor levels $N_{0}$ discussed above are not as shallow in this case and their energy distance $\left(\varepsilon_{D}\right)$ from the bottom of the conduction band is the one that the electrons are excited across above $100 \mathrm{~K}$. At higher Sb concentration, the energy gap between the donor levels and the conduction band increases further and the $p$-to- $n$ transition temperature is expected to appear at higher temperatures (Fig. 13). The extreme Sb-end member exhibits thermally activated $p$-type conduction at room temperature with no evidence of donor levels.

\section{CONCLUDING REMARKS}

Physical insights concerning structure-property relationships in the $\mathrm{K}_{2} \mathrm{Bi}_{8-x} \mathrm{Sb}_{x} \mathrm{Se}_{13}$ series were obtained from studies of charge transport properties. Increasing incorporation of $\mathrm{Sb}$ in $\mathrm{K}_{2} \mathrm{Bi}_{8} \mathrm{Se}_{13}$ leads to an enhancement of the semiconducting character and a gradual conversion from $n$ - to $p$-type transport. The property evolution is not monotonic with composition due to the nonuniform $\mathrm{Bi} / \mathrm{Sb} / \mathrm{K}$ substitution and distribution.

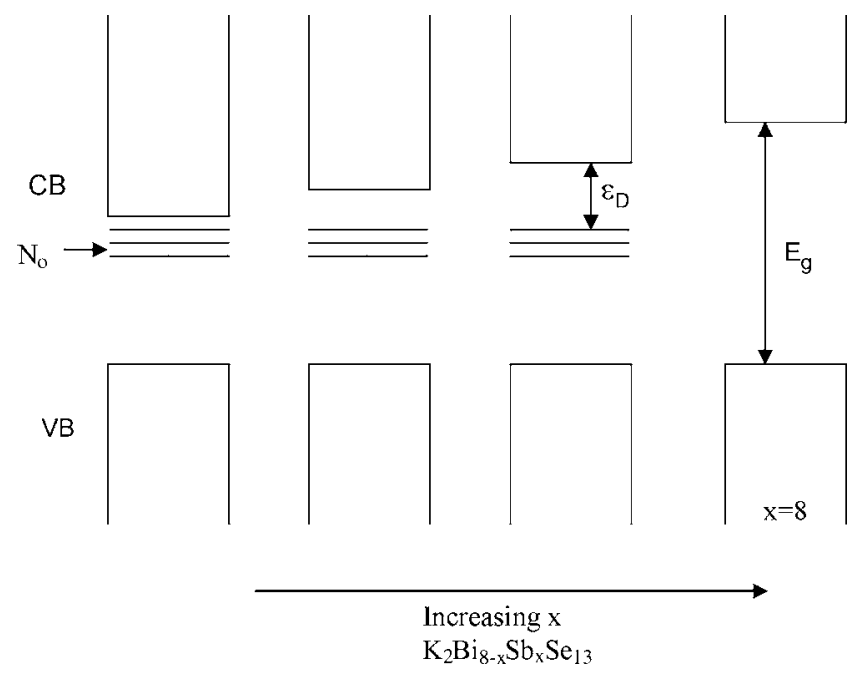

FIG. 13. Schematic of the relative position of the donor states $N_{0}$ in the band structure of $\mathrm{K}_{2} \mathrm{Bi}_{8-x} \mathrm{Sb}_{x} \mathrm{Se}_{13}$ as a function of $x$. The greater the $\mathrm{Sb}$ fraction the higher the position of the conduction band (CB) and the larger the energy separation of the donor states $N_{0}$. The top of the valence band (VB) remains relatively intact as it is dominated to Se $p$ orbitals.

The transport in these materials particularly for $x<5.6$ can be understood in the context of coexisting semimetallic and semiconducting domains in the crystal. On the Bi-rich side, a finite-gap semiconductor model, with the addition of a significant concentration of shallow donors $\left(N_{0}\right)$, which remain ionized even at low temperatures accounts for the free carrier behavior. The origin of the $N_{0}$ donors seems to be associated with degree of order or disorder at the mixed occupancy $M / \mathrm{K}$ sites. The coexistence of the $M / \mathrm{K}$ disorder and long range ordering on the $M / \mathrm{K}$ sites which was shown by electron diffraction is responsible for the observed charge transport properties of these materials. Therefore careful control of the growth conditions may reduce the semimetallic contributions and permit a significant enhancement of the thermoelectric properties through the change of the degree of disorder at the $\mathrm{K} / M$ sites.

When the content of $\mathrm{Sb}$ increases toward the Sb-rich end of the series, the disorder is reduced and finally eliminated in end compound $\mathrm{K}_{2} \mathrm{Sb}_{8} \mathrm{Se}_{13}$. Beyond the normal increase of the band gap with $\mathrm{Sb}$ incorporation, the gradual elimination of the disorder reveals a progressively more semiconducting character of $\mathrm{K}_{2} \mathrm{Bi}_{8-x} \mathrm{Sb}_{x} \mathrm{Se}_{13}$. As the $\mathrm{N}_{0}$ source of free electrons is depleted, the $p$ character is strongly enhanced.

The $n$ - to $p$-type evolution with rising $\mathrm{Sb}$ fraction in the series $\mathrm{K}_{2} \mathrm{Bi}_{8-x} \mathrm{Sb}_{x} \mathrm{Se}_{13}$ occurs at the boundary of compositional regions $\mathrm{B}$ and $\mathrm{C}$. This seems to be a critical region in which the system changes transport behavior and is of great interest for future studies. It needs to be further investigated by studying compounds with $x \sim 5.5$. It is clear that materials in this region exhibit enormous thermopower and have the potential to produce a high power factor $\left(\sigma S^{2}\right)$ at high temperature.

\section{ACKNOWLEDGMENTS}

Financial support from the Office of Naval Research is gratefully acknowledged. We thank Professor S. D. Mahanti for fruitful discussions and valuable insight during the preparation of this manuscript. 
${ }^{1}$ G. S. Nolas, J. Poon, and M. G. Kanatzidis, MRS Bull. 31, 199 (2006). ${ }^{2}$ B. Wolfing, C. Kloc, J. Teubner, and E. Bucher, Phys. Rev. Lett. 86, 4350 (2001); J. W. Sharp, B. C. Sales, D. G. Maudrus, and B. C. Chakovmakos, Appl. Phys. Lett. 74, 3794 (1999); K. Kurosaki, A. Kosuga, H. Muta, M. Uno, and S. Yamanaka, ibid. 87, 061919 (2005).

${ }^{3}$ K. F. Hsu et al., Science 303, 818 (2004); M. G. Kanatzidis, Acc. Chem. Res. 38, 359 (2005); J. Androulakis, K. F. Hsu, R. Pcionek, H. Kong, C. Uher, J. J. Dangelo, A. Downey, and M. G. Kanatzidis, Adv. Mater (Weinheim, Ger.) 18, 1170 (2006); P. F. P. Poudeu, J. D’Angelo, A. D. Downey, J. L. Short, T. P. Hogan, and M. G. Kanatzidis, Angew. Chem., Int. Ed. 45, 1 (2006)

${ }^{4}$ G. S. Nolas, M. Kaeser, R. T. Littleton, and T. M. Tritt, Appl. Phys. Lett. 77, 1855 (2000); Y. Miyazaki, X. Y. Huang, and T. Kajitani, J. Solid State Chem. 178, 2973 (2005); J. Y. Son, B. G. Kim, and J. H. Cho, Appl. Phys. Lett. 86, 221918 (2005).

${ }^{5}$ T. Caillat, A. Borshchevsky, and J. P. Fleurial, J. Appl. Phys. 80, 4442 (1996); J. Y. Yang, Y. H. Chen, W. Zhu, J. Y. Penoz, S. Q. Bao, X. A. Fan, and X. K. Duan, J. Solid State Chem. 179, 212 (2006); G. A. Lamberton et al., J. Appl. Phys. 97, 113715 (2005).

${ }^{6}$ C. Uher, J. Yang, S. Hu, D. T. Morelli, and G. P. Meisner, Phys. Rev. B 59, 8615 (1999); S. J. Poon, Semicond. Semimetals 70, 37 (2001); S. R. Culp, S. J. Poon, N. Hickman, T. M. Tritt, and J. Blumm, Appl. Phys. Lett. 88, 042106 (2006).

${ }^{7}$ K. Fujita, T. Mochida, and K. Nakamura, Jpn. J. Appl. Phys., Part 1 40, 4644 (2001); S. Lambert, H. Leligny, and D. Grebille, J. Solid State Chem. 160, 322 (2001); I. Terasaki, Y. Ishii, D. Tanaka, K. Takahata, and Y. Iguchi, Jpn. J. Appl. Phys., Part 2 40, L65 (2001).

${ }^{8}$ J. Martin, S. Erickson, G. S. Nolas, P. Alboni, T. M. Tritt, and J. Yang, J. Appl. Phys. 99, 044903 (2006); N. L. Okamoto, M. W. Oh, T. Nishii, K. Tanaka, and H. Inui, ibid. 99, 033513 (2006); A. Saramat et al., ibid. 99, 023708 (2006).

${ }^{9}$ M. G. Kanatzidis, Semicond. Semimetals 69, 51 (2000); D.-Y. Chung, L. Iordanidis, K.-S. Choi, and M. G. Kanatzidis, Bull. Korean Chem. Soc. 19, 1283 (1998); M. G. Kanatzidis, in New Thermoelectric Materials Workshop Chemistry, Physics and Materials Science of Thermoelectric Materials: Beyond Bismuth Telluride, Fundamental Materials Science Series (Kluwer Academic/Plenum, NY, 2003).

${ }^{10}$ D. Y. Chung et al., Chem. Mater. 9, 3060 (1997); T. J. McCarthy, S.-P. Ngeyi, J.-H. Liao, D. C. DeGroot, J. Schindler, C. R. Kannewurf, and M. G. Kanatzidis, ibid. 5, 331 (1993).

${ }^{11}$ Th. Kyratsi, J. S. Dyck, W. Chen, D.-Y. Chung, C. Uher, K. M. Paraskevopoulos, and M. G. Kanatzidis, Mater. Res. Soc. Symp. Proc. 691, G13.2.1 (2002); J. R. Ireland, Th. Kyratsi, M. G. Kanatzidis, and C. R. Kannewurf, ibid. 691, G13.4.1 (2002); Th. Kyratsi et al., Mater. Res. Soc. Symp. Proc. 793, 359 (2004); Th. Kyratsi, D.-Y. Chung, J. S. Dyck, C. Uher, E. Hatzikraniotis, K. M. Paraskevopoulos, and M. G. Kanatzidis, Proceedings of the Second European Conference on Thermoelectrics of the European Thermoelectric Society Poland, Kraków, 15-17 September 2004.

${ }^{12}$ D. Bilc, S. D. Mahanti, Th. Kyratsi, D.-Y. Chung, P. Larson, and M. G. Kanatzidis, Phys. Rev. B 71, 085116 (2005).

${ }^{13}$ N. F. Mott and H. Jones, The Theory of the Properties of Metals and Alloys (Dover, New York, NY, 1958).
${ }^{14}$ G. A. Slack, in CRC Handbook of Thermoelectrics, edited by D. Rowe (CRC, Boca Raton, FL, 1995), pp. 407-440; in Solid State Physics, edited by H. Ehrenreich, F. Seitz, and D. Turnbull (Academic, New York, 1997), Vol. 34, p. 1.

${ }^{15}$ J. F. Meng, N. V. Chandra Shekar, D.-Y. Chung, M. G. Kanatzidis, and J. V. Badding, J. Appl. Phys. 94, 4485 (2003).

${ }^{16}$ Th. Kyratsi, J. S. Dyck, W. Chen, D.-Y. Chung, C. Uher, K. M. Paraskevopoulos, and M. G. Kanatzidis, J. Appl. Phys. 92, 965 (2002).

${ }^{17}$ Th. Kyratsi and M. G. Kanatzidis, Z. Anorg. Allg. Chem. 629, 12, 2222 (2003).

${ }^{18}$ Th. Kyratsi, D.-Y. Chung, J. R. Ireland, C. R. Kannewurf, and M. G. Kanatzidis, Chem. Mater. 15, 3035 (2003).

${ }^{19}$ Th. Kyratsi, D.-Y. Chung, and M. G. Kanatzidis, J. Alloys Compd. 338, 36 (2002).

${ }^{20}$ Th. Kyratsi, E. Hatzikraniotis, K. M. Paraskevopoulos, J. S. Dyck, W. Chen, C. Uher, and M. G. Kanatzidis, J. Appl. Phys. 95, 4140 (2004).

${ }^{21}$ Th. Kyratsi, D.-Y. Chung, K.-S. Choi, J. S. Dyck, W. Chen, C. Uher, and M. G. Kanatzidis, Mater. Res. Soc. Symp. Proc. 626, Z8.8.1 (2000).

${ }^{22}$ CERIUS, $^{2}$ version 2.35, Molecular Simulations Inc., Cambridge, UK, 1995.

${ }^{23}$ R. B. Roberts, Philos. Mag. 36, 91 (1977).

${ }^{24} \mathrm{~A}$. Krost, $V_{x}-V I_{y}$, compounds, in Landolt-Borstein, New Series, Group III, Vol. 17f, edited by O. Madelung (Springer-Verlag, Berlin, 1983), p. 269.

${ }^{25}$ P. Lostak, I. Klichova, P. Svanda, and J. Sramkova, Cryst. Res. Technol. 34, 995 (1999).

${ }^{26}$ K. Chrissafis, Ph.D. dissertation, Aristotle University of Thessaloniki, 1993.

${ }^{27}$ J. Horak, Z. Stary, P. Lostak, and J. Pancir, J. Phys. Chem. Solids 51, 1353 (1990).

${ }^{28}$ P. Lostak, L. Benes, S. Civis, and H. Suessmann, J. Mater. Sci. 25, 277 (1990).

${ }^{29}$ A. Kalampokis, E. Hatzikraniotis, and K. M. Paraskevopoulos, Mater. Res. Bull. 33, 1359 (1998).

${ }^{30}$ E. Hatzikraniotis, Th. Kyratsi, K. Chrissafis, and K. M. Paraskevopoulos, Mater. Res. Soc. Symp. Proc. 545, 149 (1998).

${ }^{31}$ B. M. Askerov, Electron Transport Phenomena in Semiconductors (World Scientific, Singapore, 1994).

${ }^{32}$ D. Bilc, P. Larson, S. D. Mahanti, and M. G. Kanatzidis, Mater. Res. Soc. Symp. Proc. 691, G13.2.1 (2002).

${ }^{33}$ D. Bilc, P. Larson, S. D. Mahanti, and M. G. Kanatzidis, Mater. Res. Soc. Symp. Proc. 793, S6.5. (2003).

${ }^{34} \mathrm{X}$-ray diffraction measurements on these specimens do not show these supercell Bragg reflections because they are extremely weak.

${ }^{35}$ Limited crystallographic data in the $\mathrm{Sb}$-rich regions do not allow defining more accurate limits for the detailed $\mathrm{Sb} / \mathrm{Bi}$ substitution.

${ }^{36} m_{p}>m_{N}$ is the most likely case, as suggested from our calculations and those reported in Ref. 4. The effective masses $m_{N}$ and $m_{P}$ are not known for any of the $\mathrm{K}_{2} \mathrm{Bi}_{8-x} \mathrm{Sb}_{x} \mathrm{Se}_{13}$ members.

${ }^{37}$ Recent electron diffraction studies on $\beta-\mathrm{K}_{2} \mathrm{Bi}_{8} \mathrm{Se}_{13}$ indicate weak but clear evidence of a superlattice along the monoclinic $b$ axis consistent with a doubling of the unit cell (i.e., case 2). C. Malliakas and M. G. Kanatzidis (unpublished).

${ }^{38}$ Th. Kyratsi, D. Y. Chung, and M. G. Kanatzidis (unpublished). 